\title{
Antileishmanial, Antimicrobial and Antifungal Activities of Some New Aryl Azomethines
}

\author{
Yasser M.S.A. Al-Kahraman ${ }^{1}$, Hassan. M.F. Madkour ${ }^{1,2}$, Dildar Ali ${ }^{1}$ and Masoom Yasinzai ${ }^{1, *}$ \\ 1 Institute of Biochemistry, University of Balochistan, Quetta, Pakistan \\ 2 Synthetic Organic Chemistry Lab., Chemistry Department, Faculty of Science, Ain Shams \\ University, Cairo, Egypt \\ * Author to whom correspondence should be addressed; E-Mail: yasinzai_masom@yahoo.com.
}

Received: 5 December 2009; in revised form: 28 December 2009 / Accepted: 8 January 2010 / Published: 28 January 2010

\begin{abstract}
A series of eighteen azomethines has been synthesized by the reaction of appropriate primary aromatic amines with aryl and/or heteroaryl carboxaldehydes. The synthesized azomethines have been evaluated for their in vitro antileishmanial, antibacterial and antifungal activities. The results revealed some antifungal activity of most of the synthesized compounds, whereas the antileishmaniasis activity results highlighted that all synthesized azomethines inhibited parasite growth and most of them showed highly potent action towards Leishmania major promastigotes. No remarkable bactericidal activities were observed.
\end{abstract}

Keywords: azomethines; primary aromatic amines; hetaryl carboxyladehyes; antileishmanial activity; antifungal activity; antibacterial activity

\section{Introduction}

Although the last century has been characterized by a drastic lowering in the mortality caused by infectious diseases, some diseases still represent a dreadful menace to human health and therefore, for a more efficient control, require the steady development of new, more powerful and inexpensive drugs. Leischmaniasis, considered a serious public health concern in many countries, is one of these diseases. It is now endemic in many parts of Pakistan and Balochistan and the Upper Sindh are vulnerable to cutaneous leishmaniasis. The World Health Organization (WHO) has identified leishmaniasis as a 
major and increasing public health problem [1]. In spite of the social and economic importance of leishmaniasis as a tropical infectious disease, efforts directed towards the discovery of new drugs against it remain undeveloped [2,3]. Moreover, the drugs currently in use are expensive, require longterm treatment [4], display high liver and heart toxicities, develop clinical resistance after few weeks of treatment and currently contribute to increase leishmaniasis-AIDS co-infections in some countries $[5,6]$. Azomethines are characterized by the $-\mathrm{CH}=\mathrm{N}$ - (imino group) which has special importance in elucidating the mechanism of transmination and racemization in biological system [7-9]. Azomethines have high potential chemical permutation possibilities and show diuretic [10], anticancer [11-14], antibacterial [15-19], and antifungal activities [20-22]. This class of compounds has also exhibited activity against a wide range of organisms and is known to have medicinal importance and is used in drug design [23-25]. It has been also reported that when some aldehydes were functionalized by condensation with various amines, the resulting azomethines had antiparasitic activities [26].

\section{Results and Discussion}

The present work is a continuation of our ongoing programme devoted to the utilization of simple and readily obtainable materials in the synthesis of different biologically active compounds [27-33], aiming at the same time to seek a cure for the cutaneous antileishmaniasis facing our society in Pakistan by presenting some cheap azomethines that might be useful in antileishmanial and antifungal drug development.

Reaction of various substituted anilines with aromatic and/or heterocyclic aldehydes in hot methanol containing a catalytic amount of glacial acetic acid afforded the target azomethines 1-18 in good yields (55-87\%). (cf. Scheme 1).

The structural features of the synthesized azomethines were determined from the corresponding IR, ${ }^{1} \mathrm{H}-\mathrm{NMR},{ }^{13} \mathrm{C}-\mathrm{NMR}$ and mass spectroscopy data, listed in Tables 1-4. All compounds showed in the IR spectra a strong absorption band at 1,592-1,637 $\mathrm{cm}^{-1}$, typical of the stretching vibrations of the $\mathrm{C}=\mathrm{N}$ functionality, as well as the bands corresponding to $\mathrm{C}-\mathrm{H}$ stretching vibrations.

The ${ }^{1} \mathrm{H}-\mathrm{NMR}$ spectra of compounds 1-18 contained multiple signals in the $\delta 6.90$ to $8.08 \mathrm{ppm}$ regions due to aromatic protons and a singlet at 8.43-9.75 ppm from the $\mathrm{C}-\mathrm{H}$ protons of the $\mathrm{CH}=\mathrm{N}$ groups. In the spectra of compounds 5, 6, 11, 12, 13 and 14, signals between $\delta 3.50$ and 4.0 ppm were attributed to $-\mathrm{OCH}_{3}$ groups. The signals at $\delta 2.36$ and $2.33 \mathrm{ppm}$ which appeared as singlets in compounds 15 and 16 were assigned for methyl groups. In the DEPT spectra of compounds 1-18, there are no peaks at 189.0-196.0 ppm due to CHO group and the observed peaks were shifted to $\sim 160.0$ $163.0 \mathrm{ppm}$ which were attributable to $\mathrm{HC}=\mathrm{N}$ group .

Scheme 1. General synthetic route.

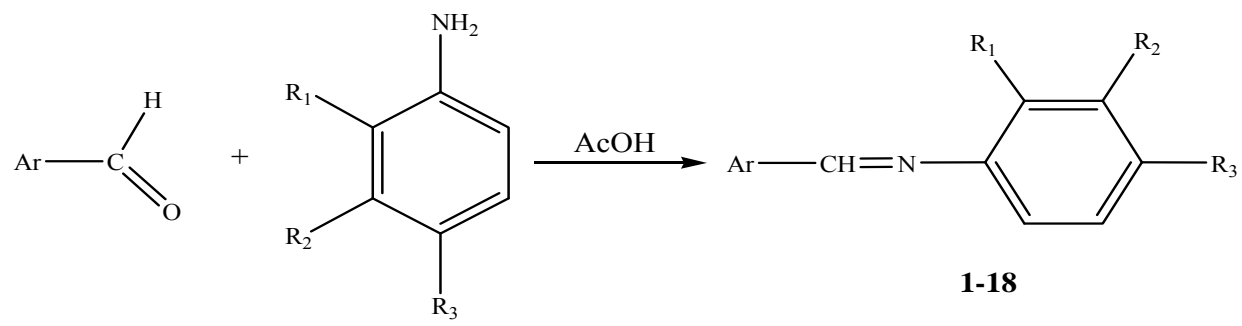


Scheme 1. Cont.

\begin{tabular}{|c|c|c|c|c|}
\hline Compd. No. & Ar & $\mathbf{R}_{1}$ & $\mathbf{R}_{2}$ & $\mathbf{R}_{3}$ \\
\hline 1 & $\mathrm{C}_{6} \mathrm{H}_{4}(\mathrm{Cl})-4$ & $\mathrm{H}$ & $\mathrm{H}$ & $\mathrm{H}$ \\
\hline 2 & $\mathrm{C}_{6} \mathrm{H}_{4}(\mathrm{Cl})-4$ & $\mathrm{H}$ & $\mathrm{H}$ & $\mathrm{NO}_{2}$ \\
\hline 3 & & $\mathrm{H}$ & $\mathrm{H}$ & $\mathrm{H}$ \\
\hline 4 & & $\mathrm{H}$ & $\mathrm{H}$ & $\mathrm{NO}_{2}$ \\
\hline 5 & $\mathrm{C}_{6} \mathrm{H}_{3}(\mathrm{OH})\left(\mathrm{OCH}_{3}\right)-3,4$ & $\mathrm{H}$ & $\mathrm{H}$ & $\mathrm{NO}_{2}$ \\
\hline 6 & $\mathrm{C}_{6} \mathrm{H}_{3}(\mathrm{OH})\left(\mathrm{OCH}_{3}\right)-3,4$ & $\mathrm{H}$ & $\mathrm{H}$ & $\mathrm{H}$ \\
\hline 7 & $\mathrm{C}_{6} \mathrm{H}_{5} \mathrm{CH}=\mathrm{CH}-$ & $\mathrm{H}$ & $\mathrm{H}$ & $\mathrm{NO}_{2}$ \\
\hline 8 & $\mathrm{C}_{6} \mathrm{H}_{5} \mathrm{CH}=\mathrm{CH}-$ & $\mathrm{H}$ & $\mathrm{H}$ & $\mathrm{H}$ \\
\hline 9 & $\mathrm{C}_{6} \mathrm{H}_{4}(\mathrm{OH})-2$ & $\mathrm{H}$ & $\mathrm{H}$ & $\mathrm{NO}_{2}$ \\
\hline 10 & $\mathrm{C}_{6} \mathrm{H}_{4}(\mathrm{Cl})-4$ & $-\mathrm{CH}=\mathrm{CH}-\mathrm{CH}=\mathrm{CH}-$ & $\mathrm{H}$ & \\
\hline 11 & $\mathrm{C}_{6} \mathrm{H}_{3}(\mathrm{OH})\left(\mathrm{OCH}_{3}\right)-3,4$ & $-\mathrm{CH}=\mathrm{CH}-\mathrm{CH}=\mathrm{CH}-$ & $\mathrm{H}$ & \\
\hline 12 & $\mathrm{C}_{6} \mathrm{H}_{4}(\mathrm{Cl})-4$ & $\mathrm{H}$ & $\mathrm{H}$ & $\mathrm{OCH}_{3}$ \\
\hline 13 & $\mathrm{C}_{6} \mathrm{H}_{3}(\mathrm{OH})\left(\mathrm{OCH}_{3}\right)-3,4$ & $\mathrm{H}$ & $\mathrm{H}$ & $\mathrm{OCH}_{3}$ \\
\hline 14 & $\mathrm{C}_{6} \mathrm{H}_{4}(\mathrm{OH})-2$ & $\mathrm{H}$ & $\mathrm{H}$ & $\mathrm{OCH}_{3}$ \\
\hline 15 & $\mathrm{C}_{6} \mathrm{H}_{4}(\mathrm{Cl})-4$ & $\mathrm{H}$ & $\mathrm{H}$ & $\mathrm{CH}_{3}$ \\
\hline 16 & $\mathrm{C}_{6} \mathrm{H}_{4}(\mathrm{OH})-2$ & $\mathrm{H}$ & $\mathrm{H}$ & $\mathrm{CH}_{3}$ \\
\hline 17 & $\mathrm{C}_{6} \mathrm{H}_{5} \mathrm{CH}=\mathrm{CH}-$ & $\mathrm{H}$ & $\mathrm{H}$ & $\mathrm{CH}_{3}$ \\
\hline 18 & & $\mathrm{H}$ & $\mathrm{H}$ & $\mathrm{NO}_{2}$ \\
\hline
\end{tabular}

Table 1. Physical and select IR data of the synthesized azomethines 1-18.

\begin{tabular}{|c|c|c|c|c|c|c|c|}
\hline \multirow[b]{2}{*}{ Compd. } & \multirow[b]{2}{*}{ Color } & \multirow{2}{*}{$\begin{array}{c}\text { Molecular }^{\mathrm{a}} \\
\text { formula } \\
\text { (Mol. Mass) }\end{array}$} & \multirow{2}{*}{$\begin{array}{l}\text { Mp }\left({ }^{\circ} \mathrm{C}\right) \\
\text { (recryst. } \\
\text { solvent) }\end{array}$} & \multirow{2}{*}{$\begin{array}{l}\text { Yield } \\
(\%)\end{array}$} & \multicolumn{3}{|c|}{ IR $\left(\mathrm{cm}^{-1}\right)$} \\
\hline & & & & & $\mathbf{N}=\mathbf{C}$ & $\begin{array}{c}\text { Azomethine } \\
\text { C-H }\end{array}$ & Substituent \\
\hline 1 & $\begin{array}{l}\text { Pale } \\
\text { yellow }\end{array}$ & $\begin{array}{c}\mathrm{C}_{13} \mathrm{H}_{10} \mathrm{ClN} \text { [34] } \\
(215.05)\end{array}$ & $\begin{array}{l}72-4 \\
\text { (L.P) } \\
\end{array}$ & 82 & 1621 & $\begin{array}{r}2874, \\
1352 \\
\end{array}$ & - \\
\hline 2 & Yellow & $\begin{array}{c}\mathrm{C}_{13} \mathrm{H}_{9} \mathrm{ClN}_{2} \mathrm{O}_{2}[35] \\
(260.04)\end{array}$ & $\begin{array}{c}140-42 \\
\text { (L.P) }\end{array}$ & 74 & 1625 & 1333 & $\begin{array}{c}1505, \\
1376\left(\mathrm{NO}_{2}\right)\end{array}$ \\
\hline 3 & Brown & $\begin{array}{c}\mathrm{C}_{11} \mathrm{H}_{9} \mathrm{NO}[36] \\
(171.07)\end{array}$ & $\begin{array}{c}106 \mathrm{~d} \\
(\mathrm{~B})\end{array}$ & 79 & 1605 & 2850,1328 & $3360(\mathrm{OH})$ \\
\hline 4 & Brown & $\begin{array}{c}\mathrm{C}_{11} \mathrm{H}_{8} \mathrm{~N}_{2} \mathrm{O}_{3}[37] \\
(216.05)\end{array}$ & $\begin{array}{l}196-98 \\
(\mathrm{EtOH})\end{array}$ & 68 & 1592 & 1285 & $\begin{array}{c}1535 \\
1339\left(\mathrm{NO}_{2}\right)\end{array}$ \\
\hline 5 & $\begin{array}{l}\text { Pale } \\
\text { orange }\end{array}$ & $\begin{array}{c}\mathrm{C}_{14} \mathrm{H}_{12} \mathrm{~N}_{2} \mathrm{OH} \\
(272.08)\end{array}$ & $\begin{array}{c}148-50 \\
(\mathrm{~L} . \mathrm{P})\end{array}$ & 64 & 1628 & 1325 & $\begin{array}{c}3354-3480(\mathrm{OH}) \\
1544,1342 \\
\left(\mathrm{NO}_{2}\right) \\
\end{array}$ \\
\hline 6 & $\begin{array}{l}\text { Pale } \\
\text { yellow }\end{array}$ & $\begin{array}{c}\mathrm{C}_{14} \mathrm{H}_{13} \mathrm{NO}_{2} \\
(227.09)\end{array}$ & $\begin{array}{c}155-57 \\
\text { (L.P) }\end{array}$ & 72 & 1621 & 1388 & $\begin{array}{c}3088 \\
\text { broad }(\mathrm{OH})\end{array}$ \\
\hline 7 & Orange & $\begin{array}{c}\mathrm{C}_{15} \mathrm{H}_{12} \mathrm{~N}_{2} \mathrm{O}_{2} \\
(252.09)\end{array}$ & $\begin{array}{c}133 \mathrm{~d} \\
(\mathrm{MeOH})\end{array}$ & 65 & 1592 & 1300 & $\begin{array}{c}1512 \\
1335\left(\mathrm{NO}_{2}\right)\end{array}$ \\
\hline
\end{tabular}


Table 1. Cont.

\begin{tabular}{|c|c|c|c|c|c|c|c|}
\hline 8 & $\begin{array}{l}\text { Pale } \\
\text { brown }\end{array}$ & $\begin{array}{l}\mathrm{C}_{15} \mathrm{H}_{13} \mathrm{~N} \\
(207.10)\end{array}$ & $\begin{array}{c}100-02 \\
(\mathrm{MeOH})\end{array}$ & 66 & 1623 & 1375 & - \\
\hline 9 & Orange & $\begin{array}{c}\mathrm{C}_{13} \mathrm{H}_{10} \mathrm{~N}_{2} \mathrm{O}_{3} \\
(242.07)\end{array}$ & $\begin{array}{c}162-64 \\
(\mathrm{~L} . \mathrm{P})\end{array}$ & 59 & 1632 & 1338 & $\begin{array}{c}1562,1347 \\
\left(\mathrm{NO}_{2}\right), 3150 \\
(\mathrm{OH})\end{array}$ \\
\hline 10 & $\begin{array}{l}\text { Pale } \\
\text { brown }\end{array}$ & $\begin{array}{c}\mathrm{C}_{17} \mathrm{H}_{12} \mathrm{ClN} \\
(265.07) \\
\end{array}$ & $\begin{array}{c}107-09 \\
(\mathrm{MeOH})\end{array}$ & 78 & 1637 & 1391 & - \\
\hline 11 & Brown & $\begin{array}{c}\mathrm{C}_{18} \mathrm{H}_{15} \mathrm{NO}_{2} \\
(277.11)\end{array}$ & $\begin{array}{l}114-16 \\
\text { (B) }\end{array}$ & 76 & 1621 & 1381 & $3048(\mathrm{OH})$ \\
\hline 12 & $\begin{array}{l}\text { Pale } \\
\text { Brown }\end{array}$ & $\begin{array}{c}\mathrm{C}_{14} \mathrm{H}_{12} \mathrm{ClNO} \\
(245.06)\end{array}$ & $\begin{array}{c}136-37 \\
(\mathrm{MeOH})\end{array}$ & 87 & 1629 & 2838,1364 & $\begin{array}{c}2838,2895 \\
\left(\mathrm{CH}_{3}\right)\end{array}$ \\
\hline 13 & $\begin{array}{l}\text { Pale } \\
\text { Brown }\end{array}$ & $\begin{array}{c}\mathrm{C}_{15} \mathrm{H}_{15} \mathrm{NO}_{3} \\
(257.11)\end{array}$ & $\begin{array}{c}257-59 \\
(\mathrm{MeOH})\end{array}$ & 70 & 1621 & 2954,1378 & $\begin{array}{l}3000 \text { (broad } \\
\mathrm{OH}), 2954, \\
2982\left(\mathrm{CH}_{3}\right)\end{array}$ \\
\hline 14 & $\begin{array}{l}\text { Greenish } \\
\text { White }\end{array}$ & $\begin{array}{c}\mathrm{C}_{14} \mathrm{H}_{13} \mathrm{NO}_{2} \\
(227.09)\end{array}$ & $\begin{array}{c}85-87 \\
(\mathrm{MeOH})\end{array}$ & 67 & 1637 & 2964,1362 & $\begin{array}{c}3610(\mathrm{OH}) \\
2964,2996 \\
\left(\mathrm{CH}_{3}\right)\end{array}$ \\
\hline 15 & White & $\begin{array}{c}\mathrm{C}_{14} \mathrm{H}_{12} \mathrm{ClN} \\
(229.07)\end{array}$ & $\begin{array}{c}127-29 \\
(\mathrm{MeOH})\end{array}$ & 79 & 1622 & 2929,1354 & $\begin{array}{c}2854,2925 \\
\left(\mathrm{CH}_{3}\right)\end{array}$ \\
\hline 16 & White & $\begin{array}{c}\mathrm{C}_{14} \mathrm{H}_{13} \mathrm{NO} \\
(211.10)\end{array}$ & $\begin{array}{c}96-98 \\
(\mathrm{MeOH}) \\
\end{array}$ & 67 & 1615 & 2920,1366 & $\begin{array}{l}3100(\mathrm{OH}), \\
2920\left(\mathrm{CH}_{3}\right)\end{array}$ \\
\hline 17 & $\begin{array}{l}\text { Pale } \\
\text { Orange }\end{array}$ & $\begin{array}{c}\mathrm{C}_{16} \mathrm{H}_{15} \mathrm{~N}[38] \\
(221.12)\end{array}$ & $\begin{array}{c}76-77 \\
(\mathrm{MeOH})\end{array}$ & 71 & 1627 & 2912,1379 & $2912\left(\mathrm{CH}_{3}\right)$ \\
\hline 18 & $\begin{array}{l}\text { Pale } \\
\text { Yellow }\end{array}$ & $\begin{array}{c}\mathrm{C}_{11} \mathrm{H}_{8} \mathrm{~N}_{2} \mathrm{O}_{2} \mathrm{~S} \text { [39] } \\
(232.03)\end{array}$ & $\begin{array}{c}135-37 \\
(\mathrm{MeOH})\end{array}$ & 55 & 1612 & 1323 & $\begin{array}{c}1543,1341 \\
\left(\mathrm{NO}_{2}\right)\end{array}$ \\
\hline
\end{tabular}

${ }^{a}$ references are given for known products, the others are new; ${ }^{b}$ L.P $=$ Light Petroleum b.p. $60-80$ ${ }^{\circ} \mathrm{C}, \mathrm{B}=$ benzene, $\mathrm{EtOH}=$ ethanol, $\mathrm{MeOH}=$ methanol.

Table 2. ${ }^{1} \mathrm{H}-\mathrm{NMR}$ of azomethines 5-16 $\left(\mathrm{DMSO}^{-} \mathrm{d}_{6}\right)(\delta$, ppm).

\begin{tabular}{|c|c|}
\hline Compd. & ${ }^{1} \mathrm{H}-\mathrm{NMR}$ \\
\hline 5 & $\begin{array}{l}9.45(\mathrm{~s}, 1 \mathrm{H},-\mathrm{OH}, \text { exchangeable }), 8.36(\mathrm{~s}, 1 \mathrm{H}, \text { benzylidenimine } \mathrm{H}), 8.08-6.90(\mathrm{~m}, 7 \mathrm{H}, \mathrm{Ar}-\mathrm{H}), \\
3.80\left(\mathrm{~s}, 3 \mathrm{H},-\mathrm{OCH}_{3}\right) .\end{array}$ \\
\hline 6 & $\begin{array}{l}9.75(\mathrm{~s}, 1 \mathrm{H},-\mathrm{OH}, \text { exchangeable), } 8.43(\mathrm{~s}, 1 \mathrm{H},-\mathrm{CH}=\mathrm{N}), 7.52-6.88(\mathrm{~m}, 8 \mathrm{H}, \mathrm{Ar}-\mathrm{H}), 3.84(\mathrm{~s}, 3 \mathrm{H}, \\
\left.-\mathrm{OCH}_{3}\right) .\end{array}$ \\
\hline 7 & $8.10(\mathrm{~m}, 1 \mathrm{H},-\mathrm{CH}=\mathrm{N}), 7.60-6.95(\mathrm{~m}, 11 \mathrm{H}, \mathrm{Ar}-\mathrm{H}$ and olefinic $\mathrm{H})$. \\
\hline 8 & $8.25(\mathrm{~d}, 1 \mathrm{H}$, benzylideimin $\mathrm{H}, J=5.36 \mathrm{~Hz}), 7.54-7.07(\mathrm{~m}, 12 \mathrm{H}, \mathrm{Ar}-\mathrm{H}$ and olefinic $\mathrm{H})$. \\
\hline 9 & $12.27(\mathrm{~s}, 1 \mathrm{H}, \mathrm{OH}$, exchangeable), $8.99(\mathrm{~s}, 1 \mathrm{H},-\mathrm{CH}=\mathrm{N}), 8.32-6.98(\mathrm{~m}, 7 \mathrm{H}, \mathrm{Ar}-\mathrm{H})$. \\
\hline 10 & $8.73(\mathrm{~s}, 1 \mathrm{H}$, benzylidenimine $\mathrm{H}), 8.09-7.23(\mathrm{~m}, 11 \mathrm{H}, \mathrm{Ar}-\mathrm{H})$ \\
\hline 11 & $\begin{array}{l}9.76(\mathrm{~s}, 1 \mathrm{H}, \mathrm{OH}, \text { exchangeable }), 8.53(\mathrm{~s}, 1 \mathrm{H}, \text { benzylidenimine } \mathrm{H}), 8.29-6.92(\mathrm{~m}, 10 \mathrm{H}, \mathrm{Ar}-\mathrm{H}), \\
3.89\left(\mathrm{~s}, 3 \mathrm{H},-\mathrm{OCH}_{3}\right) .\end{array}$ \\
\hline 12 & $8.64(\mathrm{~s}, 1 \mathrm{H},-\mathrm{CH}=\mathrm{N}), 7.93-6.97(\mathrm{~m}, 8 \mathrm{H}, \mathrm{Ar}-\mathrm{H}), 3.77\left(\mathrm{~s}, 3 \mathrm{H},-\mathrm{OCH}_{3}\right)$. \\
\hline 13 & $\begin{array}{l}9.60(\mathrm{~s}, 1 \mathrm{H}, \mathrm{OH}, \text { exchangeable }), 8.44(\mathrm{~s}, 1 \mathrm{H},-\mathrm{CH}=\mathrm{N}), 7.49-6.86(\mathrm{~m}, 7 \mathrm{H}, \mathrm{Ar}-\mathrm{H}), 3.83 \text { and } 3.76 \\
\left(2 \mathrm{~s}, 6 \mathrm{H}, 2-\mathrm{OCH}_{3}\right) .\end{array}$ \\
\hline
\end{tabular}


Table 2. Cont.

\begin{tabular}{|c|l|}
\hline $\mathbf{1 4}$ & $\begin{array}{l}13.38(\mathrm{~s}, 1 \mathrm{H}, \mathrm{OH}, \text { exchangeable), } 8.59(\mathrm{~s}, 1 \mathrm{H}, \text { benzylidenimine } \mathrm{H}), 7.36-6.89(\mathrm{~m}, 7 \mathrm{H}, \mathrm{Ar}-\mathrm{H}), \\
3.82\left(\mathrm{~s}, 3 \mathrm{H},-\mathrm{OCH}_{3}\right)\end{array}$ \\
\hline $\mathbf{1 5}$ & $8.41(\mathrm{~s}, 1 \mathrm{H},-\mathrm{CH}=\mathrm{N}), 7.83-7.11(\mathrm{~m}, 8 \mathrm{H}, \mathrm{Ar}-\mathrm{H}), 2.36\left(\mathrm{~s}, 3 \mathrm{H},-\mathrm{CH}_{3}\right)$ \\
\hline $\mathbf{1 6}$ & $\begin{array}{l}13.19(\mathrm{~s}, 1 \mathrm{H}, \mathrm{OH}, \text { exchangeable }), 8.94(\mathrm{~s}, 1 \mathrm{H},-\mathrm{CH}=\mathrm{N}), 7.63-6.93(\mathrm{~m}, 7 \mathrm{H}, \mathrm{Ar}-\mathrm{H}), 2.33(\mathrm{~s}, 3 \mathrm{H}, \\
\left.-\mathrm{CH}_{3}\right) .\end{array}$ \\
\hline
\end{tabular}

Table 3. ${ }^{13} \mathrm{C}-\mathrm{NMR}\left(\mathrm{DMSO}_{\mathrm{d}}\right)(\mathrm{d}$, ppm) of azomethines 5-16.

\begin{tabular}{|c|c|}
\hline Compd & ${ }^{13} \mathrm{C}-\mathrm{NMR}$ \\
\hline 5 & $160.0,154.2,152.4,147.3,146.3,131.0,125.5,125.2,123.2,115.9,112.3,56.1$. \\
\hline 6 & $160.2,152.4,152.0,147.3,131.1,129.9,127.2,125.5,122.3,115.9,112.3,56.1$. \\
\hline 7 & $163.7,158.6,146.4,135.2,133.3,128.5,128.5,127.9,125.2,123.2,119.8$. \\
\hline 8 & $163.7,152.4,135.2,133.3,130.0,128.6,128.5,127.9,127.2,122.3,119.9$. \\
\hline 9 & $161.1,160.0,154.2,146.4,132.4,132.1,125.2,123.2,121.4,120.5,117.8$. \\
\hline 10 & $160.0,151.9,136.6,135.0,134.5,130.5,128.9,128.6,128.3,127.8,126.8,126.3,115.2$. \\
\hline 11 & $\begin{array}{l}160.0,152.2,151.9,147.9,147.3,135.0,131.1,128.5,128.3,127.8,126.7,126.3,125.5,115.9,115.1 \text {, } \\
112.3,56.1 \text {. }\end{array}$ \\
\hline 12 & $160.0,159.1,144.3,136.5,134.5,130.6,128.8,122.1,115.6,55.8$. \\
\hline 13 & $159.9,159.1,152.4,147.3,144.3,131.1,125.5,115.9,115.6,122.1,112.3,56.1,55.8$. \\
\hline 14 & $161.1,160.1,159.1,144.3,132.4,132.1,122.1,121.4,120.4,117.8,115.5,55.8$. \\
\hline 15 & $160.1,149.1,136.9,136.5,134.5,130.6,130.3,128.9,122.2,21.3$. \\
\hline 16 & $161.1,159.9,149.1,136.8,132.4,132.1,130.3,122.2,121.4,120.5,117.7,21.3$. \\
\hline
\end{tabular}

Table 4. Mass spectra of azomethines 5-16.

\begin{tabular}{|c|c|}
\hline Compd. & m/z (\% Relative abundance) \\
\hline 5 & $272\left(\mathrm{M}^{+}, 4.25\right), 227(2.34), 139(4.85), 138(70.25), 107$ (76.60), 92 (48.80), 91 (6.91), 65 (100.00). \\
\hline 6 & $227\left(\mathrm{M}^{+}, 98.26\right), 211(21.66), 166(14.45), 154$ (7.12), $139(5.12), 104(22.42), 77(100.00)$ \\
\hline 7 & $\begin{array}{l}252\left(\mathrm{M}^{+}, 27.28\right), 251\left(\mathrm{M}-17^{+}, 83.41\right), 205 \text { (56.73), } 178 \text { (4.88), } 149 \text { (4.88), } 138 \text { (54.55), } 115 \text { (70.16), } 92 \\
(48.50), 91(47.95), 65(100.00)\end{array}$ \\
\hline 8 & $207\left(\mathrm{M}^{+}, 33.52\right), 206\left(\mathrm{M}-17^{+}, 100.00\right), 178$ (3.25), 128 (13.57), 115 (18.72), 77 (90.33). \\
\hline 9 & $242\left(\mathrm{M}^{+}, 96.65\right), 212(13.38), 195(37.11), 167$ (29.72), 151 (5.91), $120(44.48), 76(100.00)$ \\
\hline 10 & $\begin{array}{l}\left.267\left(\mathrm{M}+27^{+}, 20.42\right), 266(\mathrm{M}+1\rceil^{+}, 20.79\right), 265\left(\mathrm{M}^{+}, 60.68\right), 230(2.89), 202(3.48), 154(23.00), 127 \\
(100.00), 77(29.47), 51(9.14) .\end{array}$ \\
\hline 11 & $\begin{array}{l}277\left(\mathrm{M}^{+}, 100.00\right), 261 \text { (7.13), } 216 \text { (6.07), } 204 \text { (5.90), } 178 \text { (3.99), } 154 \text { (19.97), } 127 \text { (80.53), } 101 \text { (11.97), } \\
85 \text { (44.01), } 83 \text { (69.57), } 77 \text { (27.60). }\end{array}$ \\
\hline 12 & $\begin{array}{l}247\left(\mathrm{M}+27^{+}, 26.19\right), 246\left(\mathrm{M}+17^{+}, 15.23\right), 245\left(\mathrm{M}^{+}, 77.99\right), 230 \text { (100.00), } 201 \text { (10.17), } 167 \text { (27.80), } 149 \\
(5.64), 139(10.67), 114(4.26), 77(35.29), 63(45.11) .\end{array}$ \\
\hline 13 & $\begin{array}{l}257\left(\mathrm{M}^{+}, 100.00\right), 242 \text { (85.72), } 227 \text { (3.73), } 198 \text { (5.24), } 170 \text { (15.75), } 154 \text { (7.33), } 134 \text { (6.16), } 115 \text { (7.02), } 77 \\
(4.02), 64 \text { (19.33). }\end{array}$ \\
\hline 14 & $\begin{array}{l}228\left(\mathrm{M}+17^{+}, 16.62\right), 227\left(\mathrm{M}^{+}, 100.00\right), 212 \text { (71.79), } 183 \text { (4.33), } 154 \text { (3.40), } 128 \text { (5.33), } 105 \text { (3.08), } 77 \\
(55.04), 65(38.15) .\end{array}$ \\
\hline 15 & $\begin{array}{l}231\left(\mathrm{M}+27^{+}, 30.49\right), 230\left(\mathrm{M}+17^{+}, 33.41\right), 229\left(\mathrm{M}^{+}, 90.60\right), 193(2.89), 165(4.57), 150(2.05), 118 \\
(22.99), 91(100.00), 77(9.79), 65(61.60) .\end{array}$ \\
\hline 16 & $211\left(\mathrm{M}^{+}, 100.00\right), 210\left(\mathrm{M}-17^{+}, 76.87\right), 167$ (6.29), 120 (12.86), 118 (8.68), 91 (80.65), 65 (74.49). \\
\hline
\end{tabular}




\section{Antileishmaniasis activity}

Antileishmanial activity of the compounds (1-18) was assayed by Zhai's method [40] using a preestablished culture of L. major (Table 5) Parasites were cultured in medium M199 with 10\% foetal bovine serum; $25 \mathrm{mM}$ of HEPES, and $0.22 \mu \mathrm{g}$ of penicillin and streptomycin, respectively, at $24{ }^{\circ} \mathrm{C}$ in a shaking incubator. Compounds 1, 6 and $\mathbf{1 6}$ have moderate activity against $L$ major. Azomethines 4, 10, 14, 15, and 17 show good activity against $L$ major and azomethines 9 and 12 show significant activity. The high in vitro antileishmaniasis activity of these compounds makes them promising leads for development of effective therapeutic agents.

Table 5. \% Inhibition of azomethines against L. major leishmania.

\begin{tabular}{|c|c|}
\hline Compound & L. major $^{a}$ \\
\hline 1 & $0.77 \pm 0.09$ \\
\hline 2 & - \\
\hline 3 & - \\
\hline 4 & $0.65 \pm 0.01$ \\
\hline 5 & - \\
\hline 6 & $0.73 \pm 0.16$ \\
\hline 7 & - \\
\hline 8 & - \\
\hline 9 & $0.59 \pm 0.07$ \\
\hline 10 & $0.61 \pm 0.27$ \\
\hline 11 & - \\
\hline 12 & $0.57 \pm 0.11$ \\
\hline 13 & $0.63 \pm 0.04$ \\
\hline 14 & $0.68 \pm 0.12$ \\
\hline 15 & $0.66 \pm 0.18$ \\
\hline 16 & $0.81 \pm 0.03$ \\
\hline 17 & $0.62 \pm 0.08$ \\
\hline 18 & - \\
\hline \multicolumn{2}{|c|}{ Standard Drug $\mathrm{IC}_{50}(\mu \mathrm{g} / \mathrm{mL} \pm$ S.D $)$} \\
\hline Amphotericin B & $0.56 \pm 0.20$ \\
\hline
\end{tabular}

${ }^{a}$ percentage inhibition activity: $100=$ (non-significant; $0.95-0.80=$ low; $0.79-0.70=$ Moderate; 0.69-0.60 = Good; below 0.59-0.56 = Significant activity)

\section{Antifungal activity}

The antifungal bioassay is screened by the Agar Tube Dilution Method [41-42], in which the test compounds were screened for activity against the following organisms: Candida albicans, Aspergillus flavus, Microsportum canis, Fusarium solani and Candida glabrata. Table 6 shows \% inhibition of the synthesized azomethines. In all tests the linear growth in control was $100 \mathrm{~mm}$. It is evident from the antifungal screening that:

(i) None of the compounds are inactive against C. albicans or C. glabrata.

(ii) Only compound $\mathbf{1 7}$ has moderate activity against $A$. flavus. 
(iii) Azomethines 4, 5, 12 and 14 have low activity against $M$. canis, whereas compound 9 has good antifungal activity against the same fungus.

(iv) Azomethine 9 shows moderate activity against $F$. solani, whereas azomethines 4, 11, 17 and 18 have low activity against the same fungus.

Table 6. \% inhibition of azomethines against different types of fungi.

\begin{tabular}{|c|c|c|c|c|c|}
\hline Compound & C. albicans & A. flavus & M. canis & F. solani & C. glabrata \\
\hline $\mathbf{1}$ & 0 & 0 & 0 & 0 & 0 \\
$\mathbf{2}$ & 0 & 0 & 0 & 0 & 0 \\
$\mathbf{3}$ & 0 & 0 & 0 & 0 & 0 \\
$\mathbf{4}$ & 0 & 0 & $20^{\mathrm{a}}$ & 35 & 0 \\
$\mathbf{5}$ & 0 & 0 & 30 & 0 & 0 \\
$\mathbf{6}$ & 0 & 0 & 0 & 0 & 0 \\
$\mathbf{7}$ & 0 & 0 & 0 & 0 & 0 \\
$\mathbf{8}$ & 0 & 0 & 0 & 0 & 0 \\
$\mathbf{9}$ & 0 & 0 & 60 & 40 & 0 \\
$\mathbf{1 0}$ & 0 & 0 & 0 & 0 & 0 \\
$\mathbf{1 1}$ & 0 & 0 & 0 & 35 & 0 \\
$\mathbf{1 2}$ & 0 & 0 & 35 & 0 & 0 \\
$\mathbf{1 3}$ & 0 & 0 & 0 & 0 & 0 \\
$\mathbf{1 4}$ & 0 & 0 & 20 & 0 & 0 \\
$\mathbf{1 5}$ & 0 & 0 & 0 & 0 & 0 \\
$\mathbf{1 6}$ & 0 & 0 & 0 & 0 & 0 \\
$\mathbf{1 7}$ & 0 & 40 & 0 & 15 & 0 \\
$\mathbf{1 8}$ & 0 & 0 & 0 & 20 & 0 \\
\hline \multicolumn{7}{|l|}{ Std. Drug MIC (mg/mL) } \\
\hline Miconazole & 110.8 & - & 98.4 & 73.25 & 110.28 \\
Amphotericin B & - & 20.0 & - & - & - \\
\hline
\end{tabular}

${ }^{a}$ percentage inhibition activity: 0-39 = low (non-significant); 40-59 = Moderate; 60-69 = Good; Above 70 = Significant activity.

\section{Antibacterial activity}

The antibacterial activity of the synthesized azomethines was screened by the agar well diffusion method [43-44] against Gram-negative and Gram-positive bacteria, namely Escherchia coli, Bacillus subtilis, Shigella flexen ari, Staphylococcus aureus, Pseudomonas aeruginosa and Salmonella typhi. Only compound 4 showed significant activity ( $25 \mathrm{~mm}$ inhibition zone) against $E$. coli and moderate activity (14 $\mathrm{mm}$ zone) against $B$. subtilis.

\section{Experimental}

\section{General}

All aromatic and heterocyclic aldehydes as well as primary aromatic amines were obtained commercially from the Aldrich Chemical Company. Solvents used were of analytical grade. M.p. were measured using a Stuart (UK) electric melting point apparatus. IR spectra were recorded with a Thermo 330 FTIR spectrometer. Mass spectra were measured on a MAT 312 Finnigan instrument 
operating at $70 \mathrm{eV} .{ }^{1} \mathrm{H}$ - and ${ }^{13} \mathrm{C}-\mathrm{NMR}$ spectra were recorded on a Avance 400 Bruker instrument using TMS as internal standard and DMSO- $\mathrm{d}_{6}$ as solvent.

\section{Chemistry: General procedure for synthesis of azomethines}

An equimolar mixture of aryl and/or hetaryl carboxaldehyde with selected primary aryl amine in methyl alcohol $(30 \mathrm{~mL})$ containing few drops of glacial acetic acid was heated to reflux temperature for 3-4 hours then left overnight until the product precipitated. The product was filtered off, washed with light petroleum and recrystallized from the suitable solvent [cf. Table 1]. It is worthy to mention that anhydrous dry solvents for recrystallization were essential to avoid decomposition by hydrolysis. The crystalline solid that separated out was filtered by suction using water pump and washed with light petrol to afford the desired azomethines 1-18 in pure state.

\section{Biological Screening: Antileishmaniasis Activity - Preparation of Samples}

Each compound $(1 \mathrm{mg})$ was dissolved in DMSO $(1 \mathrm{~mL})$ and Amphotercin B (1 mg) was also dissolved in DMSO $(1 \mathrm{~mL})$ as positive control. Parasites at log phase were centrifuged at 3,000 rpm for 3 minutes. Parasites were diluted in fresh culture medium to a final density of $2 \times 10^{6}$ cells $/ \mathrm{mL}$. In 96-well plates, $180 \mu \mathrm{L}$ of medium was added in different wells. Twenty $\mu \mathrm{L}$ of the compound was added in medium and serially diluted. Parasite culture $(100 \mu \mathrm{L})$ was added in all wells. Three rows were left for negative and positive controls. In the negative controls, DMSO was serially diluted in medium while the positive control contained varying concentrations of the standard antileishmanial compound Amphotercin B. The plates were incubated for 72 hours at $24{ }^{\circ} \mathrm{C}$. The culture was examined microscopically on an improved neubaur counting chamber and $\mathrm{IC}_{50}$ values of compounds possessing antileishmanial activity were calculated. All assays were run in duplicate. $\mathrm{IC}_{50}$ of samples was determined by using the Prism software.

\section{Antifungal activity}

The concentration of the tested compounds was $200 \mathrm{ug} / \mathrm{mL}$ of DMSO. A reference antifungal and DMSO were used as positive and negative control, respectively. Incubation temperature was $27^{\circ} \mathrm{C}$ and time 7 days. The inculation of fungus was carried out with a $4 \mathrm{~mm}$ diameter piece of fungus removed from a seven-day-old culture. The growth in the compound-amended media was determined by measuring linear growth $(\mathrm{mm})$ and growth inhibition calculated with reference to the negative control and calculating the $\%$ inhibition of fungal growth:

$$
\% \text { Inhibition }=100-\frac{\text { linear growth in test }(\mathrm{mm})}{\text { linear growth in control }(\mathrm{mm})} \times 100
$$

\section{Antibacterial Activity}

The concentration of tested compound was $1 \mathrm{mg} / \mathrm{mL}$ of DMSO, whereas the concentration of the reference antibacterial drug imipenem was $10 \mathrm{mg} /$ disc. Blank test showed that DMSO in the preparations of the test solutions does not affect the test organisms. 


\section{Conclusion}

Eighteen azomethines were prepared by reacting an aromatic or heterocyclic aldehyde and an aromatic primary amine compound in a protic solvent like ethanol or methanol heated to reflux temperature in presence of few drops of acetic acid as a catalyst. The acidic catalyst leads to the increase of the electrophilicity of the caronyl function of the carboxalehyde containing reactant. The results revealed some order of fungicidal activities of most of the synthesized compounds whereas the results of leishmanicidal activity highlighted that all synthesized azomethines inhibited the growth of parasite and most of them showed high potent action towards leishmania major promastigotes. No remarkable bactericidal activities have been recorded. Azomethines are characterized by the $-\mathrm{CH}=\mathrm{N}$ (imino group) which has special importance in elucidating the mechanism of transmination and racemization in biological system. Azomethines have high potential chemical permutation and show diuretic, anticancer, antibacterial, and antifungal activities. This class of compounds also exhibited activity against a wide range of organisms and are known to have medicinal importance and used in drugs design. It has been also reported that some aldehydes when were functionalized by condensation with various amines, the produced azomethines had antiparasitic activities. It is evident that Azomethine compounds 1, 6 and 16 have moderate activity against $L$ major. Azomethines 4, 10, 14, 15, and $\mathbf{1 7}$ show good activity against $L$ major and azomethine $\mathbf{9}$ and 12, shows significant activity against L.major. The high in vitro Anti leishmaniasis activity of these compounds makes them a promising lead for development of effective therapeutic agents.It is clear that the synthesize of Azomethines showed only compound 17 has moderated activity against A flavus. Azomethine 4,5,12 and 14 have low activity against M. Canis whereas compound 9 has good anti fungal activity against same fungus. Azomethine 9 shows a moderate activity against F. Solani whereas azomehtines 4,11,17 and 18 have low activity against the same fungus. In bacteria among the 18 synthesize of azomethines tested only compound 4 showed significant activity against E.coli and low activity against B. subtilis.

\section{Acknowledgments}

This work was supported by University of Balochistan, Quetta and Higher Education Commission (HEC), Islamabad, Pakistan.

\section{References}

1. Modabber, F. Tropical Disease Research Progress Leishmaniasis. In Tropical Disease Research Progress 1991-1992; World Health Organization: Geneva, Switzerland, 1993; pp. 77-87.

2. Croft, S.L. The current status of antiparasite chemotherapy. Parasitology 1997, 114, 3-15.

3. Engers, H.D.; Bergquist, R.; Modabber, F. Progress on vaccines against parasites. Dev. Biol. Stand. 1996, 87, 73-84.

4. Murray, H.W. Treatment of visceral leishmaniasis in 2004. Am. J. Trop. Med. Hyg. 2004, 71, 787-794.

5. Croft, S.L. Recent developments in the chemotherapy of leishmaniasis. Trends Pharmacol. Sci. 1988, 9, 376-381. 
6. Berman, J.D. Chemotherapy for leishmaniasis: biochemical mechanisms, clinical efficacy, and future strategies. Rev. Infect. Dis. 1988, 10, 560-586.

7. Lau, K.Y.; Mayr, A.; Cheung, K.K. Synthesis of transition metal isocyanide complexes containing hydrogen bonding sites in peripheral locations. Inorg. Chim. Acta. 1999, 285, 223-232.

8. Ispir, E.; toroglu, S.; karyaldiz, A. Synthesis, characterization, antimicrobial and genotoxic activities of new Schiff bases and their complexes. Transition Met. Chem. 2008, 33, 953-960.

9. Ispir, E.; Kurtoglu, M.; Purtas, F. Synthesis and antimicrobial activity of new Schiff bases having the $-\mathrm{SiOR}$ group $\left(\mathrm{R}=\mathrm{CH}_{3}\right.$ or $\left.\mathrm{CH}_{2} \mathrm{CH}_{3}\right)$, and their transition metal complexes. Trans. Met. Chem. 2005, 30, 1042-1047.

10. Supuran, C.T.; Barboiu, M.; Luca, C.; Pop, E.; Brewster, M.E.; Dinculescu, A. Carbonic anhydrase activators. Part 14. Syntheses of mono and bis pyridinium salt derivatives of 2 -amino5-(2-aminoethyl)- and 2-amino-5-(3-aminopropyl)-1,3,4-Thiadiazole and their interaction with isozyme II. Eur. J. Med. Chem. 1996, 31, 597-606.

11. Sharma, K.P.; Jolly, V.S.; Phatak, P. Schiff bases and their derivatives as potential anticancer agents. Ultra Scient. Phys. Sci. 1998, 10, 263-266.

12. Kuzamin, V.E.; Artemenko, A.G.; Lozytska, R.N.; Fedtchouk, A.S.; Lozitsky, V.P.; Muratov, E.N.; Mescheriakov, A.K. Investigation of anticancer activity of macrocyclic Schiff bases by means of 4D-QSAR based on simplex representation of molecular structure. SAR QSAR Environ. Res. 2005, 16, 219-230.

13. Shingare, M.S.; Ingle, D.B. Synthesis of pyrimidine Schiff bases as anticancer agents. J. Indian Chem. Soc. 1976, 53, 1036-1037.

14. Shkawat, D.R.; Sabins, S.S.; Deliwala, C.V. Potential anticancer agents, Schiff bases from $p$-(3azaspiro[5,5]undec-3-yl)benzaldehydes. Bull. Haffkine Inst. 1973, 1, 35-39.

15. More, S.V.; Dongarkhadekar, D.V.; Chavan, R.N.; Jadhav, W.N.; Bhusare, S.R.; Pawar, R.P. Synthesis and antibacterial activity of new Schiff bases, 4-thiazolidinones and 2-azetidinones. $J$. Indian Chem. Soc. 2002, 79, 768-769.

16. Bhendkar, A.K.; Vijay, K.; Raut, A.W. Synthesis of some novel Schiff bases of 2aminopyrimidine and their antimicrobial activity. Acta Ciencia India Chem. 2004, 30, 29-32.

17. Vaghasiya, Y.K.; Nair, R.S.; Baluja, M.; Chanda, S.S. Synthesis, structural determination and antibacterial activity of compounds derived from vanillin and 4-aminoantipyrine. J. Serb. Chem. Soc. 2004, 69, 991-998.

18. Vashi, K.; Naik, H.B. Synthesis of novel Schiff base and azetidinone derivatives and their antibacterial activity. Eur. J. Chem. 2004, 1, 272-276.

19. Rhodes, R.C.; Hall, H.; Beesley, S.R.; Jenkins, J.G.; Collins, D.C.; Zheng, P. Therapeutic Potentiation of the immune system by costimulatory Schiff base-forming drugs. Nature 1995, 377, 71-75.

20. Safwat, H.M.; Ragab, F.A.; Eid, N.M.; Abdel, G.M. Synthesis, anti-tumor and antimicrobial activities of 3-chloro-9- ( $p$ - $N$-substituted sulfamoylphenylaminoethylene) acridines. Egyptian J. Pharm. Sci. 1988, 29, 99-110. 
21. Mtrei, R.; Yadawe, M.; Patil, S.A. Synthesis of biologically active p-bis(amino-5-mercapto-1,2,4triazol-3-yl)benzene and its Schiff base: a new class of bis-triazole. Orient. J. Chem. 1996, 12, $101-102$.

22. Hossain, M.E.; Allam, M.N.; Begum, J.; Akbar, M.A.; Uddin, M.N.; Smith, F.E.; Hynes, R.C. The preparation, characterization, crystal structure and biological activities of some $\mathrm{Cu}$ (II) complexes of the 2-benzoyl pyridine Schiff bases of $S$-methyl-and $S$-benzyldithiocarbazate. Inorg. Chim. Acta. 1996, 249, 207-213.

23. Khan, S.A.; Siddiqui, A.A.; Shibeer, B. Analgesic activity of isatin derivatives. Asian J. Chem. 2002, 14, 1117-1118.

24. Verma, M.; Pandeya, S.N.; Singh, K.N.; Stables, J.P. Anticonvulsant activity of Schiff bases of isatin derivatives. Acta Pharm. 2004, 54, 49-56.

25. Sari, N.; Arslan, S.; Logoglu, E.; Sakiyan, L. Antibacterial Activities of some Amino acid-Schiff bases. GUJ Sci. 2003, 16, 283-288.

26. Rathelot, P.; Azas, N.; El-Kashef, H.; Delmas, F.; Di Giorgio, C.; Timon-David, P.; Maldonado, J.; Vanelle, P. 1,3-Diphenylpyrazoles: synthesis and antiparasitic activities of azomethine derivatives. Eur. J. Med. Chem. 2002, 37, 671-679.

27. Madkour, H.; Farag, A.; Ramses, S.; Ibrahiem, N. Synthesis and fungicidal activity of new imidazoles from 2-(chloromethyl)-1 H -benzimidazole. Phosphor. Sulfur Phosphorous Silicon 2006, 181, 255-265.

28. Madkour, H.M.F. Afify, A.A.E. Abdalha, A.A. Elsayed, G.A.; Salem, M.S. Synthetic utility of enaminonitrile moiety in heterocyclic synthesis: Synthesis of some new thienopyrimidines. Phosphor. Sulfur Phosphorous Silicon 2009, 184, 719-732.

29. Nofal, Z.M.; El-Zahar, M.I.; Salem, M.A.I.; Madkour, H.M.F. Synthesis and chemoprophylactic effect of novel coumarin derivatives. Egypt J. Chem. 2005, 48, 587-604.

30. Madkour, H.M.F.; Mahmoud, M.R.; Sakr, A.M.; Habashy, M.M. Synthesis and antibacterial activity of new 4H-pyrano [3,2-h] quinolines and fused derivatives. Sci. Pharm. 2001, 69, 33-52.

31. Madkour, H.M.F.; Salem, M.A.I.; Soliman, E.A.; Mahmoud, N.F.H. A facile one-pot synthesis and antibacterial activity of aziridines and thiazines from 1,3-diarylprop-2-enones. Phosphor. Sulfur Phosphorous Silicon 2001, 170, 15-28.

32. Salem, M.A.I.; Madkour, H.M.F.; Soliman, E.A.; Mahmoud, N.F.H. Synthesis of bactericides via carbon nucleophilic addition on 1,3-diarylprop-2-enones as michael acceptors. Heterocycles 2000, $53,1129-1143$.

33. Mahmoud, M.R.; Madkour, H.M.F. Reactions of 3-3-chlorophenyl-1-4-(3,4methylenedioxybenzylidene)aminophenylprop-2-enone with some nucleophiles. Synth. Commun. 1996, 26, 3799-3808.

34. Sobanov, A.A.; Zolotukhin, A.V.; Galkina, I.V.; Galkin, V.I.; Cherkasov, R.A. Kinetics and mechanism of the Pudovik reaction in the azomethine series: III. Acid-catalyzed hydrophosphorylation of imines. Russ. J. Gen. Chem. 2006, 76, 421-429.

35. Neuvonen, H.; Neuvonen, K.; Fülöp, F. Substituent cross-interaction effects on the electronic character of the $\mathrm{C}=\mathrm{N}$ bridging group in substituted benzylidene anilines - Models for molecular cores of mesogenic compounds. A ${ }^{13} \mathrm{C}-\mathrm{NMR}$ study and comparison with theoretical results. $J$. Org. Chem. 2006, 71, 3141-3148. 
36. Montalvo-Gonza'lez, R.; Ariza-Castolo, A.; Molecular structure of di-aryl-aldimines by multinuclear magnetic resonance and X-ray diffraction. J. Mol. Struct. 2003, 655, 375-389.

37. Subudhi, B.B.; Satpathy, S.; Bhatta, D.; Pradhan, D. Synthesis of some 2-(R, R')-3-(p-nitro phenyl)-thiazolidinone as potent antifungal agents. J. Teach. Res. Chem. 2005, 12, 42-45.

38. Sain, B.; Thyagarajan, G.; Sandhu, J.S. Cycloaddition reactions of meso-ionic oxazolone with cinnamaldehyde anils. Can. J. Chem. 1980, 58, 2034-2037.

39. Youssef, M.S.K. Synthesis of antimicrobial heteroaryl-substituted-1,2,3-triazolines via the reaction of diazomethane with anils and mixed azines of thiophenealdehyde and/or isatin. $J$. Chem. Technol. Biotechnol. 1981, 31, 363-367.

40. Zhai, L.; Chen, M.; Blom, J.; Teander, T.G.; Christensen, S.B.; Karazmi, A. The antileishmanial acitivity of novel oxygenated chalcones and their mechanism of action. Antimicrob. Agents Chemother. 1999, 43, 793-803.

41. Choudhary, M.I.; Dur-e-Shahwar Parveen, Z.; Jabbar, A.; Ali, I.; Atta-Ur-Rahman. Antifungal steroidal lactones from Withania coagulance. Phytochemistry 1995, 40, 1243-1246.

42. Janaki, S.; Vijayasekaram, V. Antifungal activities of aglaia roxburghiana (W \& A). Biomedicine. 1998, 18, 86-89.

43. Alves, T.M.A.; Silva, A.F.; Brandao, M.; Grandi, T.S.M.; Smania, E.F.A.; Smania, A., Jr.; Zani, C.L. Antibacterial xanthones from Kielmeyera variabilis mart. (Clusiaceae). Mem. Inst. Oswaldo. Cruz. 2000, 95, 367-373.

44. Stepanović, S.; Antić, N.; Dakić, I.; Švabić-Vlahović, M. In vitro antimicrobial activity of propolis and synergism between propolis and antimicrobial drugs. Microbiol. Res. 2003, 158, $353-357$.

Sample Availability: Samples of the compounds 1-18 are available from the authors.

(C) 2010 by the authors; licensee Molecular Diversity Preservation International, Basel, Switzerland. This article is an open-access article distributed under the terms and conditions of the Creative Commons Attribution license (http://creativecommons.org/licenses/by/3.0/). 